\title{
The role of broth enrichment in Staphylococcus aureus cultivation and transmission from the throat to newborn infants: results from the Swedish hygiene intervention and transmission of $S$. aureus study
}

\author{
S. Mernelius • S. Löfgren • P.-E. Lindgren • A. Matussek
}

Received: 12 April 2013 / Accepted: 20 June 2013 / Published online: 2 July 2013

(C) The Author(s) 2013. This article is published with open access at Springerlink.com

\begin{abstract}
Staphylococcus aureus is detected by direct plating, whereas incubation in enrichment broth prior to plating to increase the proportion of positive samples has not been fully evaluated. $S$. aureus throat colonization has been suggested to be more common than colonization of the anterior nares, but no data are available on the transmission of $S$. aureus from the throat. Swab samples were collected from the anterior nares and umbilicus from newborn infants $(n=168)$, anterior nares, throat, skin lesions, and vagina from parents $(n=332)$, and anterior nares, throat, and skin lesions from healthcare workers $(n=231)$ at three maternity wards. spa typing was used to elucidate the transmission routes of $S$. aureus. The use of enrichment broth prior to plating increased the proportion of positive samples by $46 \%$. The prevalence of S. aureus colonization in adults was $58 \%$. Throat colonization $(47 \%)$ was significantly more common than colonization in any of the other screened sites $(p<0.001)$. In total, 103 out of $168(61 \%)$ newborn infants were colonized during their hospital stay. Overall, 124 S. aureus transmissions to newborn infants were detected. Although we detected an increased risk of transmission from the nares as compared to the throat, with an odds ratio of 4.8 [ $95 \%$ confidence interval (CI) 1.8-12.7], we detected a transmission rate of $7 \%$ from the throat. We show that $S$. aureus throat colonization is more common than colonization in any of the other sites among the parents and staff. We also show evidence of transmission from the throat.
\end{abstract}

S. Mernelius · S. Löfgren · P.-E. Lindgren · A. Matussek $(\bowtie)$ Clinical Microbiology, Division of Medical Services, County Hospital Ryhov, 55185 Jönköping, Sweden

e-mail: andreas.matussek@1j.se

S. Mernelius · P.-E. Lindgren

Division of Medical Microbiology, Department of Clinical and Experimental Medicine, Linköping University, Linköping, Sweden

\section{Introduction}

The clinical spectrum of diseases caused by Staphylococcus aureus ranges from skin- and soft-tissue infections to lifethreatening invasive infections, but it is also a commensal of the human flora. Swedish guidelines for culturing samples from skin- and soft-tissue infections indicate direct plating onto solid medium, i.e., blood-, hematin-, and CLED-agar, incubated aerobically, anaerobically, and in carbon dioxide [1]. The incubation of swabs in enrichment broth prior to plating has been suggested to increase the sensitivity of $S$. aureus detection $[2,3]$. Using enrichment broth with methicillin in combination with polymerase chain reaction (PCR) increased the recovery of methicillin-resistant $S$. aureus (MRSA) by $35 \%$, compared to direct plating on selective medium [4]. The use of broth (without the addition of methicillin) prior to plating has, however, not been fully evaluated regarding increased sensitivity of methicillin-sensitive $S$. aureus (MSSA) detection. However, a recent report showed increased sensitivity from broth enrichment when used for multisite sampling of pregnant women and their neonates [5].

Approximately $30 \%$ of the population is colonized with S. aureus in the anterior nares [6], and nasal colonization poses a threat for endogenous infection $[7,8]$. Several studies have, therefore, focused on the eradication of nasal carriage of $S$. aureus to minimize the risk of endogenous infections [9-12]. The transmission of $S$. aureus from hospital staff and/or fellow patients has previously been proposed as an explanation for infections with strains not observed in the anterior nares of the patient [13]. Recent studies have indicated that throat colonization might be even more common than nasal colonization $[2,14]$. Therefore, the importance of screening for $S$. aureus carriage in the throat, as well as the 
inclusion of the throat in eradication schemes, has been discussed $[2,14,15]$. To our knowledge, no data are available on the transmission of $S$. aureus from the throat.

In this study, we aimed to clarify the effect on S. aureus detection by using an enrichment broth prior to plating compared to direct plating. Also, we determined the prevalence of $S$. aureus throat colonization and the importance of throat colonization in the transmission of $S$. aureus from healthcare workers (HCWs) and parents to newborn infants.

\section{Materials and methods}

Enrichment broth and plating of $S$. aureus samples

Swab samples were collected from the anterior nares and umbilicus from newborn infants and from the anterior nares, throat, skin lesions, and vagina (only mothers) from adults (Table 1). Swabs were streaked on BBL ${ }^{\text {TM }}$ CHROMagar ${ }^{\mathrm{TM}}$ Staph aureus medium (Becton Dickinson, Franklin Lakes, NJ) and subsequently submerged into $5 \mathrm{~mL}$ of enrichment broth, according to Nilsson and Ripa [3], except for the addition of phenyl red. Plates and broths were incubated for $18-24 \mathrm{~h}$ at $35^{\circ} \mathrm{C}$ (broth on gentle agitation). Colonies suspected to be $S$. aureus from direct plating were verified by DNase testing and replated onto BBL ${ }^{\text {TM }}$ CHROMagar ${ }^{\text {TM }}$ Staph aureus medium (Becton Dickinson). From samples that did not grow any $S$. aureus in direct plating, $10 \mu \mathrm{L}$ of broth was plated onto BBL $^{\text {TM }}$ CHROMagarTM Staph aureus medium (Becton Dickinson) and incubated at 35 C for 18-24 h. S. aureus verification was performed as described above.

\section{Throat colonization and transmission}

This study was performed at the three delivery wards in Jönköping County (Sweden) in 2009 and 2010. Parents arriving at any of the delivery wards were asked to participate. Families were excluded if a caesarean section was performed or if the newborn infant had to be treated at the intensive care unit. Written informed consent was collected from all parents, which was also valid for their underaged ( $<18$ years old) children. Oral information regarding the study was given to staff. The results from cultures were communicated to participants, if desired, by the responsible scientists.

The sampling scheme for parents $(n=332 ; 167$ mothers, 165 fathers), staff members ( $n=231 ; 91 \%$ females), infants ( $n=168 ; 83$ girls, 85 boys), visiting siblings $(n=33)$, and adult visitors $(n=2)$ has been described previously [16]. Briefly, staff members were sampled at the start of their shifts and parents were sampled upon arrival at the delivery ward. A vaginal sample was collected from the mothers at the first vaginal examination. Staff members and parents were sampled once. Infants were sampled from the anterior nares and umbilicus at $2 \mathrm{~h}$ postpartum and subsequently every $24 \mathrm{~h}$ until discharge.

S. aureus culture and verification was performed as described above.

spa typing was performed on one colony from each $S$. aureus-positive sample (either from direct plating or plating after enrichment broth), as described previously [16]. PCR products were purified and sequenced by GATC Biotech AG (Konstanz, Germany) and a spa type was assigned to each isolate using the Ridom StaphType Software (v1.5.21, Ridom GmbH, Würzburg, Germany), as described previously [17]. Based upon repeat pattern (BURP) cluster analysis was performed as described previously, using default settings [18].

Three possible transmission sources were evaluated. Primarily, the infant's own family, meaning that, if the infant's spa type was detected among its own family members, they were considered the source of transmission, even if the spa type was also present in staff and/or family members of other newborn infants. Secondarily, the staff and/or family members of other newborn infants, simultaneously cared for at the department, were considered the source of transmission if the infant's spa type was found among those but not among its own family. Finally, if the infant's spa type could not be detected either among its own family, members of staff, another family, or in the environment, the source was considered unknown.
Table 1 Samples positive for Staphylococcus aureus by direct plating and additional proportion of $S$. aureus-positive samples after broth enrichment

\begin{tabular}{lllll}
\hline & $\begin{array}{l}\text { No. of } \\
\text { samples }\end{array}$ & $\begin{array}{l}\text { Samples positive } \\
\text { (by direct plating, } n \text { ) }\end{array}$ & $\begin{array}{l}\text { Additional positive } \\
\text { samples after broth } \\
\text { enrichment, } n\end{array}$ & $\begin{array}{l}\text { Additional proportion } \\
\text { of positive samples (\%) }\end{array}$ \\
\hline Vagina & 157 & 3 & 8 & 267 \\
Nares (infants) & 524 & 38 & 47 & 124 \\
Throat & 500 & 121 & 121 & 100 \\
Skin lesions & 62 & 9 & 3 & 33 \\
Nares (adults) & 597 & 162 & 24 & 15 \\
Umbilicus & 531 & 155 & 22 & 14 \\
All sites & 2,371 & 488 & 225 & 46 \\
\hline
\end{tabular}


Table 2 Prevalence (\%) of $S$. aureus in the various sites sampled

*Also including visiting relatives and siblings

\begin{tabular}{|c|c|c|c|c|c|c|}
\hline & \multicolumn{6}{|l|}{ Culture site } \\
\hline & Anterior nares & Throat & Vagina & Skin lesions & Umbilicus & Total \\
\hline Mothers & 32 & 40 & 7 & 42 & & 51 \\
\hline Fathers & 45 & 60 & & 23 & & 70 \\
\hline Female HCWs & 31 & 43 & & 5 & & 53 \\
\hline Male HCWs & 32 & 37 & & & & 58 \\
\hline Female adults & 32 & 42 & 7 & 18 & & 53 \\
\hline Male adults & 43 & 58 & & 23 & & 69 \\
\hline All adults & 36 & 47 & 7 & 20 & & 58 \\
\hline Female infants & 43 & & & & 53 & 61 \\
\hline Male infants & 40 & & & & 53 & 61 \\
\hline All infants & 42 & & & & 53 & 61 \\
\hline All populations* & 37 & 47 & 7 & 20 & 53 & 59 \\
\hline
\end{tabular}

samples from the nares of infants and the throats of adults (Table 1).

\section{S. aureus colonization}

A total of 2,434 samples were collected from 766 individuals, resulting in $788 \mathrm{~S}$. aureus isolates, corresponding to $32 \%$ of the samples being positive for $S$. aureus.

The colonization rates are shown in Table 2. The overall prevalence of S. aureus colonization in adults was $58 \%$. Skin lesions $(n=60)$ were only sampled from adults and $20 \%$ of them contained $S$. aureus. Adult males were significantly more often colonized than females $(69 \%$ and $53 \%$, respectively, $p=0.0002$ ). In total, $61 \%$ of the newborn infants were colonized during hospitalization and no gender-associated difference was observed. At the age of $2 \mathrm{~h}, 10 \%$ and $8 \%$ of the newborn infants were colonized in the anterior nares and the umbilicus, respectively. Most of the observed colonizations occurred during the following $22 \mathrm{~h}$ of life in the umbilicus (Fig. 1).
Fig. 1 Site-specific and overall prevalence of Staphylococcus aureus among newborn infants, from age $2 \mathrm{~h}$ to discharge

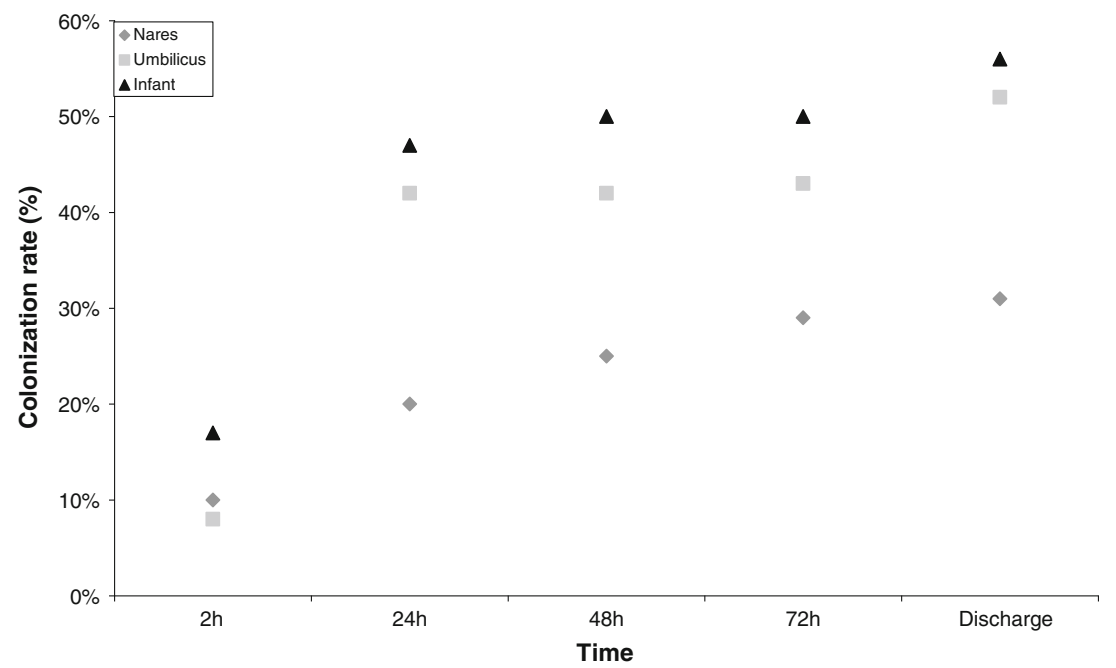


Fig. 2 spa type distribution among adults, infants, and siblings

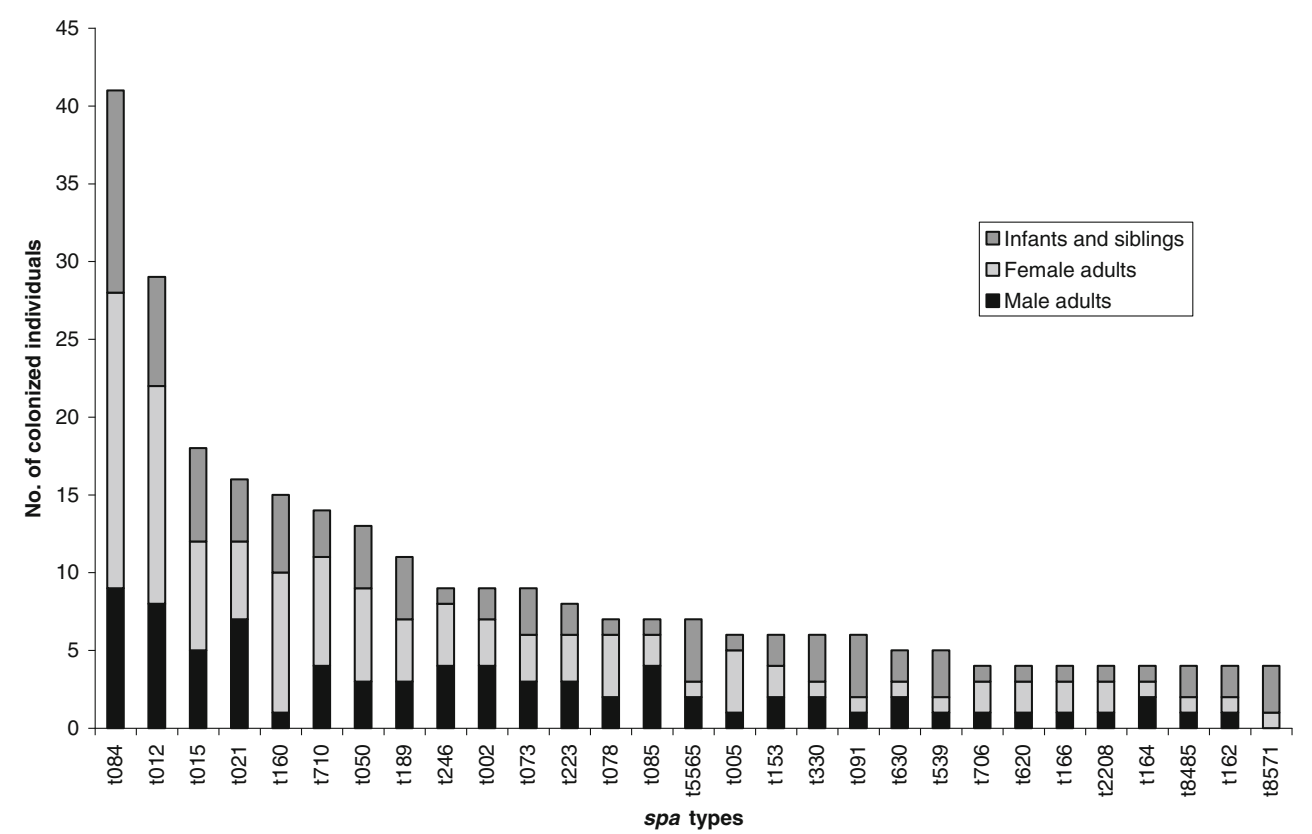

Among adults, throat colonization (47\%) was significantly more common than colonization in any of the other sites sampled $(p<0.001)$ (Table 2$)$. This was also the case when dividing the adults into females and males $(p<0.001)$. Infants were significantly more often colonized in the umbilicus $(53 \%)$ than the anterior nares $(42 \%, p<0.05)$.

spa typing and cluster analysis

In total, 170 different spa types were detected and 84 of these (49\%) were found in single individuals. The distribution of the most frequently isolated spa types is shown in Fig. 2, with spa type t084 being the most common (9\%, 74 of 788 isolates), followed by t012 (5\%). Among adults, no specific spa type was associated with female or male gender (Fig. 2), nor was there a difference in the anterior nares compared to the throat (data not shown). The spa type distribution showed a high diversity among both parents and staff; t160 was, however, more common among staff as compared to parents $(p=0.004)$. We found 140 adults that were colonized in more than one site. Of these, 42 individuals (30\%) were colonized with $S$. aureus of more than one spa type; thereby, they were defined as multiclonal individuals. Thirteen isolates $(1.6 \%)$ were considered non-typable. BURP cluster analysis revealed 19 clusters and nine singletons (12 spa types shorter than five repeats were detected and excluded from the analysis). The largest cluster was spa CC 015, including 53 (31\%) of all spa types and 297 (38\%) of all isolates, followed by spa CC 084 (including $8 \%$ of spa types and $14 \%$ of isolates), spa CC 349 (8\% of spa types and $4 \%$ of isolates), and spa CC 002 (6\% of spa types and $6 \%$ of isolates).
Transmission of S. aureus

In total, $124 \mathrm{~S}$. aureus transmissions to newborn infants were detected. The majority (57\%, 71 of 124) of these originated from a family member of the colonized infant, 21 from the mother, 19 from the father, and, for the remaining 31, the origin could be the mother, father, or another relative. In $28 \%$, the source of transmission was either a staff member or a parent of other newborn infants. In $16 \%$, no source was identified. Infants with colonized parents had a significantly increased odds ratio (OR) $\left[\mathrm{OR}_{\mathrm{MH}}\right.$ of $2.8,95 \%$ confidence interval (CI) 1.8-4.5, adjusted for gender of adult] for colonization than infants of non-colonized parents $(p<0.001)$. The rate of transmission from the anterior nares and the throat was compared and from $34 \%$ of nares-only carriers and from $7 \%$ of throat-only carriers, transmission occurred. The highest rate of transmission (61 \%) occurred from multiple-site carriers (Table 3). Thus, there is an increased

Table 3 Colonization state of possible transmitters and transmissions from these individuals

\begin{tabular}{llll}
\hline Colonization state & Number & \multicolumn{2}{l}{ Transmissions } \\
\cline { 3 - 4 } & & $n$ & $\%$ \\
\hline Multiple-site* & 102 & 62 & 61 \\
Nares only & 44 & 15 & 34 \\
Throat only & 99 & 7 & 7 \\
Total & 325 & 124 & 37 \\
\hline
\end{tabular}

*Individuals colonized with $S$. aureus of the same spa type in more than one site 
risk of transmission from the nares as compared to the throat $(\mathrm{OR}=4.8,95 \%$ CI 1.8-12.7).

\section{Discussion}

In this study, we show that the incubation of swabs in enrichment broth prior to plating increased the proportion of $S$. aureus-positive samples by $46 \%$. We also show that $S$. aureus throat colonization is more common than nasal colonization, which is in accordance with recent studies [2, 14]. Furthermore, this study also indicates the transmission of $S$. aureus originating from the throat.

We found the $S$. aureus colonization rate to be higher in all sites sampled when using enrichment broth prior to plating, with at least a doubling in positive samples from the anterior nares of infants, the throat of all populations, and vaginal samples from mothers. Thus, to determine the true rate of $S$. aureus colonization incubation in enrichment broth prior to plating seems necessary.

In this study, the highest $S$. aureus colonization rate was seen in the throat, which supports the suggestion to also include throat samples in $S$. aureus screening programs [2].

Several studies have focused on the eradication of $S$. aureus nasal colonization to prevent endogenous infections [9-11]. One study suggested that infections with strains not observed in the anterior nares of the patient might originate from hospital staff and/or fellow patients [13]. In this study, we show that the transmission potential of the anterior nares is five times higher than that of the throat. This is based on the rate of transmission when the anterior nares or the throat is the only potential source. However, as throat colonization is high and the fact that we show that transmission from the throat does occur, the eradication of throat colonization might be necessary to prevent endogenous $S$. aureus infections and nosocomial spread. This was also suggested in a recent review article [19].

We show that transmission mainly originates from the parents of the colonized infant, independent of whether it was the mother or the father who was colonized. Infants of colonized parents also had an increased risk of colonization compared to infants of non-colonized parents. One limitation of our study is that we only study transmissions to newborn infants and, therefore, we cannot draw firm conclusions regarding transmissions to adults. Furthermore, the role of broth enrichment in MRSA screening programs may differ from our data; however, increased MRSA detection rates by the use of broth enrichment has been shown previously [4].

As $91 \%$ of the HCWs are females, similar colonization rates for HCWs and mothers was expected. Colonization was more frequent in adult males as compared to adult females, which has been described previously [16]. This genderassociated difference in colonization was not observed for newborn infants. Most of the infants that were colonized before discharge were so in the umbilicus, whereas nasal colonization was much lower. Lebon et al. showed a dramatic decrease in $S$. aureus colonization rates among infants, starting at $52 \%$ at 1.5 months of age, dropping to only $13 \%$ at 14 months of age [20]. We could, in this study, see that nasal colonization in infants starts already at birth, reaching $56 \%$ at discharge, which usually occurred at 24 to $48 \mathrm{~h}$ of age.

The most common spa type in this study, t084, has recently been described as a newcomer in our geographical area [16], and it is also common in Northern Norway [21, 22]. We could not find a gender-specific spa type among adults, which has been described previously [21]. We show that spa type t160 was significantly associated with staff, as compared to a Norwegian study where t012 and t015 were associated with staff [22]. Multiclonal colonization occurred in $30 \%$ of the colonized adults. Multiclonal colonization, in both individuals and sites, could have an impact on antibiotic treatment, given simultaneous colonization with susceptible and resistant strains, and impair epidemiological conclusions. This question would, therefore, need further focus. The two largest spa clusters in our study contained more than one-third of all the spa types and more than half of the isolates. On the contrary, almost half of the spa types were only detected in single individuals.

In conclusion, we show that the use of enrichment broth for the incubation of swabs prior to plating has a high additive effect on the proportion of $S$. aureus-positive samples and that the transmission of $S$. aureus from the throat is relevant.

Acknowledgments We would like to acknowledge all of the participating families and all of the staff members at the maternity wards who have helped us in obtaining the samples. We would also like to thank Ann-Sofie Ekblom, Pia Karlsson, and Kirstin Dienus for the technical assistance. This study was financed, in part, by Futurum - the Academy for Healthcare, Jönköping County Council, and the Medical Research Council of Southeast Sweden.

Conflict of interest The authors declare that they have no conflict of interest.

Open Access This article is distributed under the terms of the Creative Commons Attribution License which permits any use, distribution, and reproduction in any medium, provided the original author(s) and the source are credited.

\section{References}

1. Bergström S, Claesson B, Hallander H, Nyberg A, Thore M, Wollin R (2003) Provsättning. In: Claesson B, Hallander H, Nyberg A, Thore M, Wollin R (eds) Referensmetodik. Bakteriologisk diagnostik av infektioner i hud, mjukdelar, skelett och inre organ, 1st edn, vol. 1. Smittskyddsinstitutet, Solna, p 134-135 
2. Wanten GJ, Schneeberger PM, Bevers A, van Ginneken E, Koolen MI (1998) Optimizing screening procedures for Staphylococcus aureus nasal carriage in patients on haemodialysis. Nephrol Dial Transplant 13:1256-1258

3. Nilsson P, Ripa T (2006) Staphylococcus aureus throat colonization is more frequent than colonization in the anterior nares. J Clin Microbiol 44:3334-3339

4. Nilsson P, Alexandersson H, Ripa T (2005) Use of broth enrichment and real-time PCR to exclude the presence of methicillinresistant Staphylococcus aureus in clinical samples: a sensitive screening approach. Clin Microbiol Infect 11:1027-1034

5. Andrews JI, Fleener DK, Messer SA, Kroeger JS, Diekema DJ (2009) Screening for Staphylococcus aureus carriage in pregnancy: usefulness of novel sampling and culture strategies. Am J Obstet Gynecol 201:396.e1-396.e5

6. Nulens E, Gould I, MacKenzie F, Deplano A, Cookson B, Alp E, Bouza E, Voss A (2005) Staphylococcus aureus carriage among participants at the 13th European Congress of Clinical Microbiology and Infectious Diseases. Eur J Clin Microbiol Infect Dis 24:145148

7. Kluytmans JA, Mouton JW, Ijzerman EP, Vandenbroucke-Grauls CM, Maat AW, Wagenvoort JH, Verbrugh HA (1995) Nasal carriage of Staphylococcus aureus as a major risk factor for wound infections after cardiac surgery. J Infect Dis 171:216-219

8. Luzar MA, Coles GA, Faller B, Slingeneyer A, Dah GD, Briat C, Wone C, Knefati Y, Kessler M, Peluso F (1990) Staphylococcus aureus nasal carriage and infection in patients on continuous ambulatory peritoneal dialysis. N Engl J Med 322:505-509

9. Kluytmans JA, Manders MJ, van Bommel E, Verbrugh H (1996) Elimination of nasal carriage of Staphylococcus aureus in hemodialysis patients. Infect Control Hosp Epidemiol 17:793-797

10. Kluytmans JA, Mouton JW, VandenBergh MF, Manders MJ, Maat AP, Wagenvoort JH, Michel MF, Verbrugh HA (1996) Reduction of surgical-site infections in cardiothoracic surgery by elimination of nasal carriage of Staphylococcus aureus. Infect Control Hosp Epidemiol 17:780-785

11. Talon D, Rouget C, Cailleaux V, Bailly P, Thouverez M, Barale F, Michel-Briand Y (1995) Nasal carriage of Staphylococcus aureus and cross-contamination in a surgical intensive care unit: efficacy of mupirocin ointment. J Hosp Infect 30:39-49

12. Bode LG, Kluytmans JA, Wertheim HF, Bogaers D, VandenbrouckeGrauls CM, Roosendaal R, Troelstra A, Box AT, Voss A, van der Tweel I, van Belkum A, Verbrugh HA, Vos MC (2010) Preventing surgical-site infections in nasal carriers of Staphylococcus aureus. N Engl J Med 362:9-17
13. Perl TM, Cullen JJ, Wenzel RP, Zimmerman MB, Pfaller MA, Sheppard D, Twombley J, French PP, Herwaldt LA; Mupirocin And The Risk Of Staphylococcus Aureus Study Team (2002) Intranasal mupirocin to prevent postoperative Staphylococcus aureus infections. N Engl J Med 346:1871-1877

14. Marshall C, Spelman D (2007) Re: is throat screening necessary to detect methicillin-resistant Staphylococcus aureus colonization in patients upon admission to an intensive care unit? J Clin Microbiol 45:3855

15. Harbarth S, Schrenzel J, Renzi G, Akakpo C, Ricou B (2007) Is throat screening necessary to detect methicillin-resistant Staphylococcus aureus colonization in patients upon admission to an intensive care unit? J Clin Microbiol 45:1072-1073

16. Mernelius S, Löfgren S, Lindgren PE, Blomberg M, Olhager E, Gunnervik C, Lenrick R, Tiefenthal Thrane M, Isaksson B, Matussek A (2013) The effect of improved compliance with hygiene guidelines on transmission of Staphylococcus aureus to newborn infants: the Swedish Hygiene Intervention and Transmission of $S$. aureus study. Am J Infect Control (in press). doi:10.1016/j.ajic.2012.09.014

17. Harmsen D, Claus H, Witte W, Rothgänger J, Claus H, Turnwald D, Vogel U (2003) Typing of methicillin-resistant Staphylococcus aure$u s$ in a university hospital setting by using novel software for spa repeat determination and database management. J Clin Microbiol 41:5442-5448

18. Mellmann A, Weniger T, Berssenbrugge C, Rothgänger J, Sammeth M, Stoye J, Harmsen D (2007) Based Upon Repeat Pattern (BURP): an algorithm to characterize the long-term evolution of Staphylococcus aureus populations based on spa polymorphisms. BMC Microbiol 7:98

19. van Rijen MM, Bonten M, Wenzel RP, Kluytmans JA (2008) Intranasal mupirocin for reduction of Staphylococcus aureus infections in surgical patients with nasal carriage: a systematic review. J Antimicrob Chemother 61:254-261

20. Lebon A, Labout JA, Verbrugh HA, Jaddoe VW, Hofman A, van Wamel W, Moll HA, van Belkum A (2008) Dynamics and determinants of Staphylococcus aureus carriage in infancy: the Generation R Study. J Clin Microbiol 46:3517-3521

21. Sangvik M, Olsen RS, Olsen K, Simonsen GS, Furberg AS, Sollid JU (2011) Age- and gender-associated Staphylococcus aureus spa types found among nasal carriers in a general population: the Tromso Staph and Skin Study. J Clin Microbiol 49:4213-4218

22. Olsen K, Sangvik M, Simonsen GS, Sollid JU, Sundsfjord A, Thune I, Furberg AS (2013) Prevalence and population structure of Staphylococcus aureus nasal carriage in healthcare workers in a general population. The Tromsø Staph and Skin Study. Epidemiol Infect 141:143-152 